\title{
CONTROLS, LLRF AND INSTRUMENTATION SYSTEMS FOR ILC TEST FACILITIES AT FERMILAB*
}

\author{
B. Chase, M. Votava, M. Wendt, FNAL, Batavia, IL 60510, U.S.A.
}

\begin{abstract}
The major controls and instrumentation systems for the ILC test areas and the NML test accelerator at Fermilab are discussed. The test areas include 3 separate areas for Vertical Superconducting RF Cavity Testing, Horizontal Cavity Testing, and NML RF and beam test area. A common control infrastructure for the test areas including a controls framework, electronic logbook and cavity database will be provided, while supporting components supplied by collaborators with diverse areas of expertise (EPICS, DOOCS, LabVIEW, and Matlab). The discussions on the instrumentation systems are focused on overview and requirements.
\end{abstract}

\section{INTRODUCTION}

A $500 \mathrm{GeV}$ center-of-mass $\mathrm{e}^{+} / \mathrm{e}^{-}$International Linear Collider (ILC) is proposed as the next generation accelerator to study the fundamental nature of matter and energy Bunches of particles are accelerated in the Main Linacs (ML) using $1.3 \mathrm{GHz}$ superconducting RF (SCRF) accelerating structures (cavities). As part of the ILC Global Design Effort (GDE), a set of SCRF infrastructure facilities for R\&D of superconducting cavities and cryomodules is currently under construction at Fermilab. In addition to the assembly halls, cleanrooms, high-power RF generation and distribution systems, these SCRF test facilities need support for (beam) instrumentation, lowlevel RF generation and control (LLRF), and an integrated data acquisition and control system. The three major ILC R\&D test areas are:

- VTS: The Vertical Test Stand for initial test of bare, so-called "undressed" SC 9-cell cavities.

- HTS: The Horizontal Test Stand for a full power test of dressed (including tuner, piezo elements, HOM couplers, field probe feedthroughs, etc.) SC cavities.

- NML: The ILC test accelerator at the New Muon Lab (NML) building will be used as final, integrated system test of complete cryomodules, including the RF system, by probing an ILC-like beam.

All three test areas need a data acquisition and control system. The sophistication and size of LLRF and instrumentation systems are small to moderate for the test stands, but substantial for the NML test accelerator.

\section{INSTRUMENTATION}

Most instrumentation activities are focused on the beam instruments, to be provided for beam operation at the NML test accelerator. However, the HTS, as well as the initial RF tests of the cryomodule in the NML facility require some "other" instrumentation activities.

*Work supported by Fermi Research Alliance, LLC under Contract No. DE-AC02-07CH11359 with the United States Department of Energy.

\section{Beam Instrumentation}

The preliminary layout of the NML beam-lines requires the following of basic beam instrumentation:

- 20x Button-style beam position monitors (BPMs) located along injector and high-energy beam-lines, with a $\sim 20 \mu \mathrm{m}$ single bunch resolution.

- $\sim 5 \mathrm{x}$ Toroidal transformers for bunch charge measurements, with better than $3 \%$ accuracy.

- 20x Screen monitors (OTR, YaG, slits, and combined function) for beam profile/emittance measurements.

The pickup detectors for most of these beam instruments can be reused from the A0-Photoinjector, but the readout systems have to be upgraded. The signal processing of the new read-out techniques is mostly realized in the digital domain, utilized by different types of in-house developed digitizers:

- 12-ch. 16-bit $5 \mathrm{MS} / \mathrm{s}$ general purpose VME digitizer.

- 16-ch. 14-bit 50 MS/s VME low-cost digitizer.

- 16 ch. 12-bit $500 \mathrm{MS} / \mathrm{s}$ digitizer, based on the high availability (HA) Advanced Telecommunications Computing Architecture (ATCA) hardware platform, used for high speed applications (BPMs).
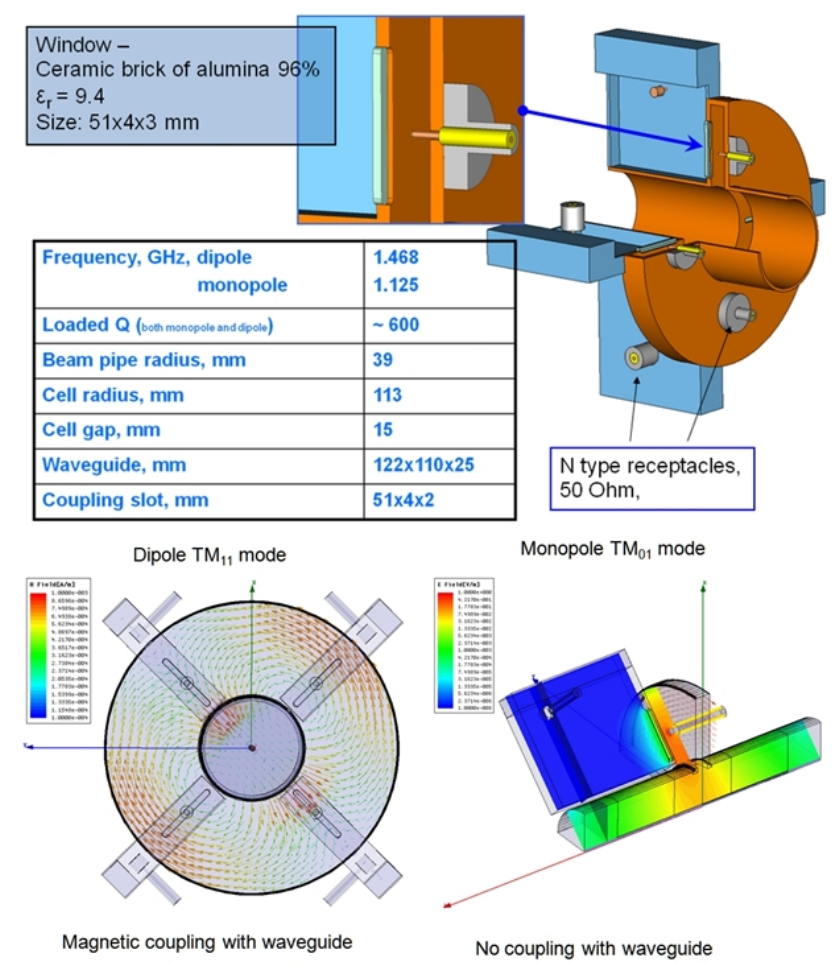

Figure 1: Cold L-Band cavity BPM. 
For the cryomodule a cold L-Band cavity BPM is currently under development[1], which will offer a $<1$ $\mu \mathrm{m}$ single bunch resolution (Fig. 1). Also the higher order mode (HOM) couplers of each SCRF cavity (a total of 16 per cryomodule) will be equipped with a dedicated readout system for beam orbit and cavity alignment studies.

Other advanced beam monitors required in the NML test accelerator are:

- 4x Bunch arrival time (or beam phase) monitors, based on electro-optical modulator techniques, promising $<50$ fs single bunch time resolution.

- Bunch length and (long.) profile monitors, based on coherent transition radiation (CTR) spectrometer techniques and electro-optical sampling methods.

A cold deflecting mode cavity (DMC) will be installed to study the longitudinal bunch profile in a separate, low energy beam-line. The high energy beam-line downstream of the cold accelerating modules offers dedicated space for further advanced beam instrumentation studies, such as LASER wire-scanners, and other electro-optical beam monitors.

\section{"Other" Instrumentation}

An RF interlock system was developed for various test stands based on a set of VME64X modules[2]. Its primary function is to protect the klystrons and input couplers by controlling the presence of the high power RF. The detection is based on:

Klystron: Forward and reflected power, presence of window arcs using PMT's, RF leakage, waveguide pressure.

Coupler: PMT, photodiode based arc detection and field emission probe (FEP) measurements, ceramic window temperature measurement.

Cavity: PMT arc detection, vacuum control, FEP HOM couplers, gradient monitoring.

The system removes the RF permit within 200-800 ns of fault detection. The new RF interlock that will be installed at NML test accelerator will receive several smaller improvements, as well as more compact board layouts for the increased number of channels.

Other instrumentation for the cryomodule, needed prior to NML beam operation includes:

Faraday cups: A Faraday cup will be installed at each end of the cryomodule's beam tube - but outside the cryogenic system - to detect the dark current caused by field emission. It will be equipped with an HV screen to suppress the emission of secondary particles, and a sensitive pre-amplifier to detect currents $<1 \mathrm{pA}$. The optional insertion of metal screens into the beam path allow an estimation of the energy distribution

Wire position monitors: The first cryomodule will be equipped with a so-called "wire position monitor" system, used to study the alignment of cavities, quadrupole and BPM, i.e. the "straightness" of the 300 mm diameter He return pipe. A RF feed signal will be supplied to the wire, and a digital receiver based system is foreseen to read-out the 7 stripline detectors in horizontal and vertical planes.
The NML test linac will accelerate an ILC-like beam; up to 3000 bunches, $5 \mathrm{~Hz}$ repetition rate, with $2 \times 10^{10}$ electrons per bunch. Using 3 cryomodules, the beam energy will reach $750 \mathrm{MeV}$, equivalent to $\sim 40 \mathrm{~kW}$ of beam power. Therefore an effective machine protection system (MPS) is mandatory, which will rely on a system of distributed beam loss monitors (BLM), based on ionization chambers, and/or photo-multiplier tubes (PMT).

\section{CONTROLS AND TIMING}

The controls systems for the three test areas originated from different evolutionary paths that will help guide the direction for the controls system development for the cryomodule beam test area at NML. Much of the VTS system in use at Fermilab has been developed as part of an MOU agreement with Jefferson Lab, including a LabVIEW based controls system. In contrast, HTS physically shares infrastructure such as cryogenics with the High Intense Neutron Source (HINS) project[3] and has been primarily developed in EPICS. Finally, the 3.9 GHz cavity R\&D effort at Fermilab, including the A0Photoinjector beam-line has been developed in close collaboration with DESY and has adopted DOOCS as the controls system. Much of this equipment, including parts of the A0-Photoinjector itself, will be moved to NML.

NML is designed to be a test facility that supports international collaborations and as such must be able to support the testing of components brought to the facility. The expertise of the our collaborators as well as the diversity of controls systems already in use in the test areas dictate that NML must support both EPICS and DOOCS at least for front end support of control and data acquisition. With the development of software that enables application level software that reads data from either type of front end, applications such as the state manager, alarm handler, and data quality analysis from several applications including DOOCS, EPICS, MatLab, and LabVIEW. All test areas will interface to two reporting packages: an electronic control room logbook (CRL[4]) which is currently deployed at the test areas and a cavity data management system under development now. Figure 2 shows an example of the overview of the controls system software at NML.

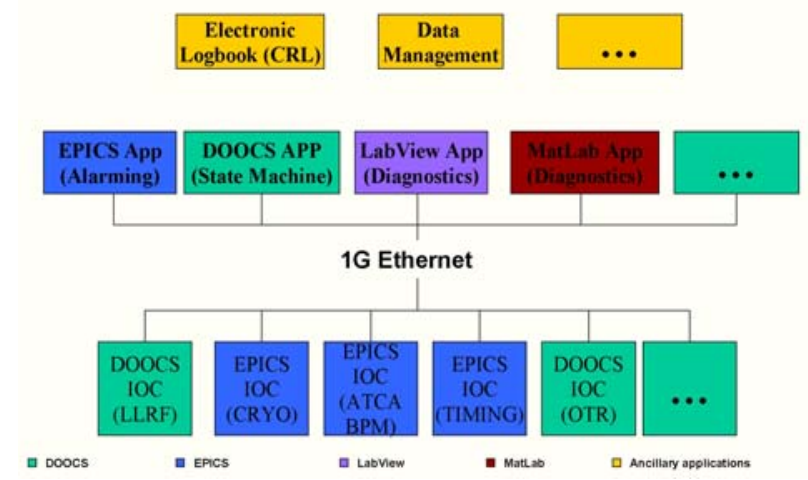




\section{FIGURE 2: EXAMPLE NML CONTROLS}

The timing system consists of three parts; an event clock generator, a rep-rate generator and clock receiver/trigger modules. ${ }^{*}$ The system is based on the Fermilab TCLK protocol that encodes eight-bit events on a carrier signal. The carrier signal is a $9 \mathrm{MHz}$ clock received from the RF master oscillator. The clock generator receives encode event triggers from a repetition rate generator that is synchronized to the AC power line. The rep-rate generator can provide rates of $10 \mathrm{~Hz}, 5 \mathrm{~Hz}$, $2 \mathrm{~Hz}, 1 \mathrm{~Hz}, 0.5 \mathrm{~Hz}, 0.2 \mathrm{~Hz}$, and $0.1 \mathrm{~Hz}$ to accommodate various modes of operation. By design, all event triggers are generated relative to a common time base and are coherent with the $10 \mathrm{~Hz}$ event.

The trigger modules consist of a clock decoder and eight independent delay timers. Each timer has an associated register for delay, output pulse width, and event reference. Additionally, each timer module provides $3 \mathrm{MHz}$, and $1 \mathrm{MHz}$ pulse trains synchronous to the $9 \mathrm{MHz}$ carrier. These modules reside in LLRF and RF Interlock VME crates and typically provide triggers for the modulator, RF, and gates for RF power interlocks. Similar clock systems are currently in use at HTS, HINS, and the A0-Photoinjector.

\section{LOW LEVEL RF (LLRF)}

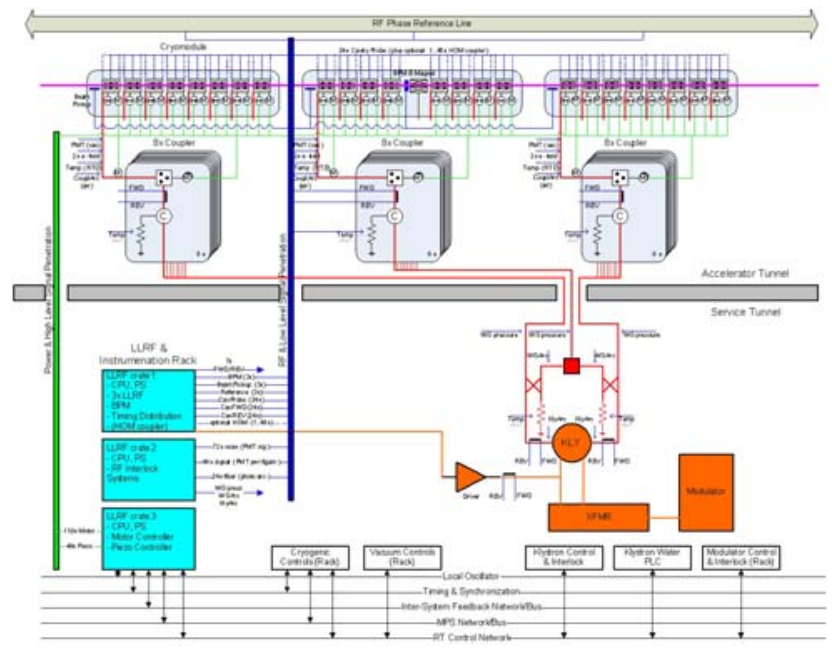

Figure 3: Low level RF and related instrumentation systems for a single ILC RF unit (3 cryomodules)

The Main Linac of the ILC will consist of 562 RF units that contain one Klystron and 26 cavities in three cryomodules. The Low Level RF Control System (Fig. 3) is responsible for delivering $0.1 \%$ beam energy regulation requirement of the Linac, which translates into 0.25 degrees phase and $0.5 \%$ amplitude field control in the buncher sections of each Linac.[5] Demonstrating this

\footnotetext{
* Implementation of a system wide synchronized timestamp scheme has yet to be addressed.
}

\section{ARCHITECTURE}

regulation performance and meeting high availability goals are important parts of the S2 milestones for the ILCTA program at Fermilab. In addition to the Test Accelerator, Low Level RF systems support the operations at VTS and HTS.

The LLRF system for VTS is a modified version of the Jlab VTS system. In a vertical test, the $\mathrm{Z}$ dimension of the cavity is not controlled as it is in a cryomodule, so the LLRF operates as a phase lock loop to maintain the drive frequency at the instantaneous resonant frequency of the cavity. Power settings and the measurements of RF power as well as automation of the procedures and cavity data analysis are implemented using custom LabVIEW virtual instruments.

The HTS LLRF is based on the DESY collaboration Simcon3.0 system. Fermilab has added to this effort with a new version of this controller with modified firmware that will be used at HTS. High-level control and GUI interfaces are available in Matlab, DOOCS and soon to be tested in EPICS. Measured cavity data will be acquired and made available in a database set up to track each cavity's parameters throughout its lifetime. HTS will be run by operators and will require increased levels of automation and self-diagnostics.

Work at NML will be staged based on the availability of cryogenics, High level RF and by the number of cryomodules in the main RF section. The first 8-cavity module will be controlled with the same type of system as is installed at HTS. As more cryomodules are added the LLRF will be upgraded to use VXI based, Multi-channel Field Controller (MFC) cards. A set of three, 32-channel MFC cards will process all the RF signals present in a full RF unit. A new receiver chassis is being designed that will downconvert up to 96 channels of $1300 \mathrm{MHz} \mathrm{RF}$ signals to the $13 \mathrm{MHz}$ IF used by the controller modules. The receiver chassis will hold up to 12, 8-channel receiver modules. Each module will also provide IF signals to the piezo resonant controller.

In order to measure the accelerator and beam parameters, a phase stable reference line will distribute the Master Oscillator signal to both the RF and instrumentation systems. This line will be a test bed for the ILC local reference. A beam energy spectrometer will independently measure cavity field regulation in the entire accelerator.

\section{REFERENCES}

[1] A. Lunin, et. al., "Design of a Submicron Resolution Cavity BPM for the ILC Main Linac ,” Proc. of the DPAC’07, Venice, Italy, May 2007.

[2] P. Prieto, to be published.

[3] http://protondriver.fnal.gov

[4] http://cepa.fnal.gov/CRL/

[5] S. Nagaitsev, "RF Phase Stability Measurements with one or two RF Units,” ILC S2 Task Force, January, 
2007 